\title{
Avaliação da contaminação microbiana em drogas vegetais
}

\section{Adriana Bugno ${ }^{1 *}$, Adriana Aparecida Buzzo ${ }^{1}$, Cristina Terumi Nakamura', Tatiana Caldas Pereira ${ }^{1}$, Dulcilena de Matos², Terezinha de Jesus Andreoli Pinto ${ }^{3}$}

${ }^{1}$ Seção de Controle de Esterilidade - Instituto Adolfo Lutz, ${ }^{2}$ Seção de Micologia - Instituto Adolfo Lutz, ${ }^{3}$ Departamento de Farmácia - Faculdade de Ciências Farmacêuticas, Universidade de São Paulo

*Correspondência:

A. Bugno

Instituto Adolfo Lutz

Seção de Controle de Esterilidade

Av. Dr. Arnaldo, 355 - Cerqueira César

01246-902 - São Paulo - SP

E-mail: adrbugno@ial.sp.gov.br
O aumento no consumo de drogas vegetais transformou seu uso em um problema de Saúde Pública, devida à possibilidade de acesso a produtos sem adequadas condições de uso. A preocupação com a qualidade é principalmente devida ao potencial de contaminação microbiana, por sua origem natural. Noventa e uma amostras compostas por sessenta e cinco espécies vegetais foram avaliadas quanto a contaminação microbiana presente. Os resultados indicaram que 93,2\% das espécies vegetais não cumpriram com os parâmetros farmacopêicos de aceitação e sugerem a necessidade de medidas regulatórias e educacionais que garantam a qualidade destes produtos.

\author{
Unitermos \\ - Drogas vegetais \\ - Controle de qualidade \\ - Contaminação \\ microbiológica
}

\section{INTRODUÇÃO}

Desde a antiguidade, plantas têm sido utilizadas como medicamentos, na prevenção, no tratamento e na cura de distúrbios, disfunções ou doenças em homens e animais (Capasso, 1986; Garcia et al., 2003; Korolkovas, 1996; Rates, 2001). Os povos primitivos iniciaram a identificação de vegetais que melhor se adequavam ao uso medicinal, da época de colheita, das técnicas de extração e de modos de conservação (Garcia et al., 2003; Korolkovas, 1996).

As descobertas de substâncias ativas em plantas medicinais impulsionaram uma revolução científica e tecnológica e os medicamentos vegetais foram sendo substituídos por fármacos sintéticos (Capasso, 1986; Korolkovas, 1996; Rates, 2001). Nas últimas décadas, porém, tem-se verificado tendência mundial de aumento na demanda por plantas e preparações de origem vegetal como recurso terapêutico, influenciado por fatores econômicos, sociais e culturais
(Abu-Irmaileh e Afifi, 2003; Bent e Ko, 2004; Calixto, 2000; Capasso, 1986; Chan, 2003; De Smet, 2004; Elvin-Lewis, 2001; Giveon et al, 2004; Mahady, 2001).

A maior industrialização e comercialização de medicamentos naturais tornaram seu uso um problema de Saúde Pública. O aumento da demanda, associado à falta de fiscalização efetiva que garanta desde a exploração racional dos recursos naturais empregados como matéria-prima, até a dispensação do produto acabado, contribuem para a disponibilidade e acesso a produtos muitas vezes sem condições adequadas ao uso, sem garantia da qualidade, segurança e eficiência, fundamentais para a recuperação ou preservação da saúde do consumidor (Bent e Ko, 2004; Calixto, 2000; Chan, 2003; De Smet, 2004; Elvin-Lewis, 2001; Giveon et al, 2004; Rates, 2001; Marques, 1996).

Fatores como poluição na água de irrigação, atmosfera, solo, condições da coleta, manipulação, secagem e estocagem são importantes a serem considerados no con- 
trole de produtos naturais, por permitirem altos níveis de contaminação microbiana, por vezes patogênica (AbouArab et al., 1999; Bermudez et al., 1983; De Smet, 2004; Fennell et al., 2004; Fischer et al., 1993, 1996; Mandeel, 2005). Ao considerar os produtos de origem vegetal com finalidade terapêutica, verifica-se a importância de especificações adequadas de qualidade microbiológica, da mesma forma que ocorre para os demais medicamentos não estéreis (Fischer et al., 1993, 1996).

O objetivo deste estudo foi investigar a contaminação microbiológica em amostras de drogas vegetais, isolando e identificando espécies contaminantes.

\section{MATERIAL E MÉTODOS}

\section{Amostras}

Foram avaliadas 91 amostras de drogas vegetais, compostas por 65 espécies vegetais distintas. As amostras foram escolhidas por sua disponibilidade comercial e popularidade de uso, tendo sido obtidas de diferentes fornecedores, na cidade de São Paulo: abutua (Chondrodendron tomentosum), agoniada (Plumeria lancifolia), alcachofra (Cynara scolymus -3 amostras), alfazema (Lavandula officinalis -2 amostras), alteia (Althaea officinalis), angélica (Angelica archangelica), aquiléia (Achillea millefolium), bardana (Arctium lappa), boldo-do-Chile (Peumus boldus - 2 amostras), calumba (Jateorhiza palmata), camédrio (Teucrium chamaedrys), camomila (Matricaria recutita - 3 amostras); capim-limão (Cymbopogon citrates); cardo-santo (Carduus benedictus); caroba (Jacaranda caroba), carqueja (Baccharis gaudichaudiana - 2 amostras), carvalho (Quecus robur), cáscara-sagrada (Rhamnus purshiana - 3 amostras), castanha-da-Índia (Aesculus hippocastanum), catuaba (Trichilia catigua - 2 amostras), cavalinha (Equisetum arvense - 2 amostras), centáurea menor (Centaurium erythraea), chá-debugre (Cordia ecalculata), chá-verde (Camellia sinensis), chapéu-de-couro (Echinodorus macrophyllus - 2 amostras), cipó-prata (Banistera argyrophylla), condurango (Marsedenia condurango), erva-doce (Pimpinella anisum), erva-mate (Illex paraguariensis), escamônea (Convolvulus scammonia), espinheira-santa (Maytenus ilicifolia), estigma de milho (Zea mays), fava tonca (Dipteryx odorata), frângula (Rhamnus frangula), fucus (Fucus vesiculosus - 3 amostras), funcho (Foeniculum vulgare), ginkgo (Ginkgo biloba - 4 amostras), guaraná (Paullinia cupana -3 amostras), hipérico (Hypericum perforatum), hissopo (Hyssopus officinalis), ipê roxo (Tabebuia avellanedae), jaborandi (Pilocarpus microphylus), jalapa (Phytolacca americana - 2 amostras), jasmim (Jasminum officinale), jurubeba (Solanum paniculatum), losna (Artemisia absinthium), macela
(Achyrocline satureoides), malva (Malva sylvestris), marapuama (Ptychopetalum olacoides), melissa (Melissa officinalis -2 amostras), pfáffia (Pfaffia paniculata), quássia (Quassia amara - 2 amostras), quebra-pedra (Phyllanthus niruri), quina amarela (Chinchona calisaya), ratânia (Krameria triandra), ruibarbo (Rheum palmatum), sabugueiro (Sambucus nigra - 3 amostras), sene (Cassia Senna - 2 amostras), stévia (Stevia rebaudiana - 2 amostras), sucupira (Bowdichia spp), tília (Tilia cordata), urucum (Bixa orellana), uva-ursi (Arctostaphylos uva-ursi), valeriana (Valeriana officinalis) e verbasco (Verbascum densiflorum).

\section{Preparação das amostras}

Porções de $10 \mathrm{~g}$ de amostra foram adicionadas a $90 \mathrm{~mL}$ de Água Peptonada Tamponada (MERCK) e homogeneizadas em agitador tipo vórtex, por 2 minutos. A partir desta diluição inicial foram executadas diluições decimais seriadas, também em Água Peptonada Tamponada, com homogeneização em agitador tipo vórtex, por 1 minuto.

\section{Enumeração de bactérias heterotróficas e de fungos}

Foi utilizada a técnica de semeadura em profundidade conforme descrito em compêndios oficiais (US Pharmacopeia, 2005), utilizando Ágar Caseína de Soja (MERCK) e Ágar Dextrose Batata (DIFCO) com cloranfenicol para enumeração de bactérias e fungos, respectivamente. As placas foram incubadas em posição invertida a $35 \pm 1{ }^{\circ} \mathrm{C}$ por 48 horas para enumeração de bactérias e a $26 \pm 1{ }^{\circ} \mathrm{C}$ por 7 dias, para fungos.

\section{Identificação de bactérias contaminantes}

A identificação de bactérias contaminantes foi executada, após a incubação da diluição inicial a $36 \pm 1{ }^{\circ} \mathrm{C}$ por 48 horas, conforme indicado em compêndios oficiais (US Pharmacopeia, 2005), abrangendo a pesquisa para Staphylococcus aureus, Salmonella spp, Escherichia coli e outras enterobactérias, Pseudomonas aeruginosa e outras bactérias Gram-negativas não fermentadoras, além da pesquisa de Bacillus cereus (Rhodehamel e Harmon, 2001).

Para a identificação, foram realizados cultivos em meios de cultura seletivos como Ágar Baird Parker (DIFCO), Ágar Vogel Johnson (DIFCO) e Ágar Manitol Salgado (DIFCO), para a pesquisa de Staphylococcus aureus; Ágar Cetrimide (MERCK), Ágar para Detecção de Piocianina (DIFCO) e para Detecção de Fluoresceína (DIFCO), para pesquisa de Pseudomonas aeruginosa; Ágar Xilose Lisina Desoxicolato, Ágar Verde Brilhante e Ágar Salmonella-Shigella, após enriquecimento seletivo 
em Caldo selenito-cistina e Caldo Rappaport-Vassiliadis, para a pesquisa de Salmonella spp; Ágar eosina azul de metileno - Levine e Ágar verde brilhante, para a pesquisa de Escherichia coli, outras enterobactérias e bactérias Gram-negativas não fermentadoras e Ágar seletivo para Bacillus cereus. A confirmação da identidade de colônias supeitas, obtidas em meios de cultura seletivos, foi executada por técnicas de coloração e provas bioquímicas.

\section{Identificação de fungos contaminantes}

O isolamento de colônias observadas nas placas de enumeração de fungos foi realizado em Ágar Dextrose Batata (MERCK), com incubação a $26 \pm 1{ }^{\circ} \mathrm{C}$ por 10 dias, sendo a identificação realizada pela observação das características macromorfológicas e micromorfológicas, seguindo esquemas taxonômicos de Raper e Fennel (1965) e Pitt (1979).

\section{RESULTADOS E DISCUSSÃO}

Foram calculadas as populações médias obtidas na enumeração de bactérias e fungos para cada uma das 65 espécies vegetais avaliadas, sendo a freqüência de distribuição destas amostras apresentada na Figura 1.

No Brasil, a Resolução RDC n ${ }^{\circ} 48$, de 16 de março de 2004 (Brasil, 2004), estabelece que a pesquisa de contaminantes microbiológicos em fitoterápicos deve estar de acordo com especificações farmacopêicas. Tanto a Farmacopéia Brasileira (1988), quanto a Farmacopéia
Americana (2005) estabelecem as seguintes especificações para produtos de uso oral: $10^{3}$ bactérias aeróbias $/ \mathrm{g}$ ou $\mathrm{mL}$, $10^{2}$ fungos/g e ausência de Salmonella spp, Escherichia coli e Staphylococcus aureus. No entanto, a Farmacopéia Brasileira (1988) indica também a pesquisa de outros indicadores de maior risco para a via de administração oral, como Pseudomonas aeruginosa, Bacillus cereus, Enterobacter spp, Candida albicans, Aspergillus flavus e Aspergillus parasiticus.

Observando-se os resultados obtidos, verificou-se que $58,5 \%$ das drogas vegetais analisadas apresentaram populações de bactérias superiores a $10^{3} / \mathrm{g}$ e $63,1 \%$, populações de fungos superiores a $10^{2} / \mathrm{g}$.

Em relação à contaminação por bactérias heterotróficas, Czech et al. (2001) avaliaram a contaminação microbiana em 131 espécies vegetais comercialmente disponíveis na Áustria e Alemanha e detectaram populações médias superiores a $10^{3} \mathrm{UFC} / \mathrm{g}$ em todas as amostras. Outros estudos da qualidade microbiológica de drogas vegetais e preparações derivadas, como aqueles realizados por Alexander et al. (1997), que avaliaram produtos disponíveis no mercado inglês, por Kneifel e Berger (1994), que avaliaram produtos do mercado austríaco, e por Fischer et al. (1993), que avaliaram produtos disponíveis no Brasil, obtiveram popuações superiores a $2 \times 10^{3} \mathrm{UFC} / \mathrm{g}$ em $52,7 \%$, $94,5 \%$ e $70,3 \%$ das amostras analisadas, respectivamente, enquanto Santos et al. (1995), no Brasil e Bermudez et al. (1983), na Espanha, detectaram populações desta ordem em apenas $33,3 \%$ e $26,3 \%$ das amostras analisadas.

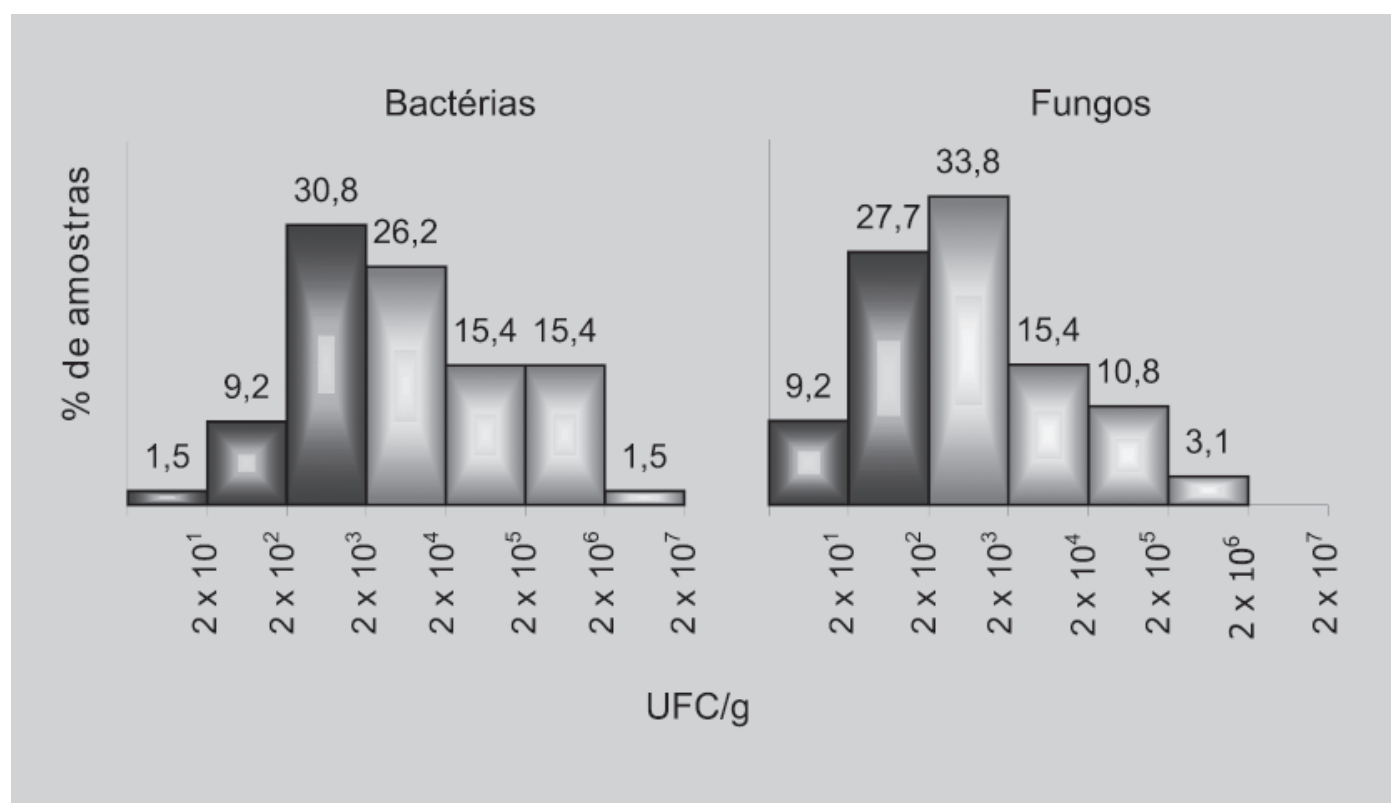

FIGURA 1 - Freqüência de distribuição das amostras de drogas vegetais de acordo com a população de microrganismos contaminantes. 
Em relação à população fúngica, Bermudez et al (1983) detectaram populações médias superiores a $10^{12}$ $\mathrm{UFC} / \mathrm{g}$ em todas as amostras de matérias-primas naturais analisadas; populações superiores a $10^{2} \mathrm{UFC} / \mathrm{g}$ foram detectadas em $93,5 \%$ das amostras de chás avaliadas por Martins et al. (2001), em Portugal, e em 100\% das amostras analisadas por El-Kady et al. (1992), no Egito, e por Halt (1998), na Croácia. Czech et al. (2001), Garrido et al. (1992) na Espanha e Lutomski e Kedzia (1980) na Polônia, detectaram estas populações fúngicas em $90,3 \%$, $87,5 \%$ e $90 \%$ das amostras, respectivamente.

Cinqüenta e um tipos de drogas vegetais avaliadas apresentaram populações microbianas superiores aos limites farmacopêicos estabelecidos, sendo que 19,6\% destas amostras apresentaram populações superiores a $10^{3}$ bactérias/g (altéia, angélica, cardo-santo, chá-verde, erva-mate, fava tonka, fucus, ginkgo e quássia), $25,5 \%$, populações superiores a $10^{2}$ fungos/g (abutua, agoniada, aquiléia, boldo-do-Chile, calumba, caroba, castanha-da-India, centáurea, chá-de-bugre, cipó-prata, espinheira santa, ipêroxo e marapuama) e $54,9 \%$, populações superiores para bactérias e fungos (alcachofra, camédrio, camomila, capimlimão, carqueja, catuaba, cavalinha, chapéu-de-couro, condurango, erva-doce, estigma de milho, guaraná, hipérico, hissopo, jaborandi, jalapa, jasmim, losna, macela, malva, melissa, pfáffia, quebra-pedra, ratânia, sene, stévia, tília e verbasco). Elevadas cargas microbianas são indicativas da possibilidade de ocorrência de microrganismos patogênicos ou potencialmente patogênicos.

Os resultados obtidos na pesquisa e identificação de microrganismos específicos, apresentados na Figura 2, indicam que os microrganismos Salmonella spp e
Staphylococcus aureus não foram detectados nas drogas vegetais analisadas, embora tenham sido detectados Staphylococcus spp em 3,1\% das espécies vegetais, enquanto Pseudomonas aeruginosa foi detectada em apenas uma espécie vegetal (1,5\%). Estes resultados estão em conformidade com dados obtidos por Alexander et al. (1997) com amostras obtidas no mercado da Inglaterra, e por Santos et al. (1995), com amostras obtidas no mercado brasileiro, que verificaram baixa ocorrência de Pseudomonas aeruginosa e ausência de Salmonella spp e Staphylococcus aureus; por Czech et al. (2001), com amostras do mercado austríaco e alemão, e por Kneifel e Berger (1994), com amostras obtidas também no mercado austríaco, que detectaram apenas Staphylococcus spp coagulase positiva em $0,7 \%$ e em $5 \%$ das amostras analisadas, respectivamente.

Embora os estudos de Alexander et al. (1997) e de Czech et al. (2001) tenham verificado baixa ocorrência de Escherichia coli (2\% e 2,9\%, respectivamente), neste estudo este microrganismo foi detectado em $26,2 \%$ das espécies vegetais. A presença de Enterobacter spp e Klebsiella spp foi verificada em $52,3 \%$ e $60,0 \%$ das espécies vegetais, respectivamente, sendo as ocorrências superiores às obtidas por Alexander et al. (1997), que detectaram Enterobacter spp e Klebsiella spp em 29,5\% e em 3\% das amostras, e por Santos et al. (1995), que detectaram Enterobacter spp em 37,2\% das amostras.

A presença de Bacillus cereus foi verificada em 9,2\% das espécies vegetais, resultado próximo aos obtidos por Bermudez et al. (1983) e por Santos et al. (1995), que detectaram este microrganismo, respectivamente, em $6,0 \%$ e 3,9\% das amostras. Por outro lado, Kneifel e Berger (1994), Czech et al. (2001) e Martins et al. (2001)

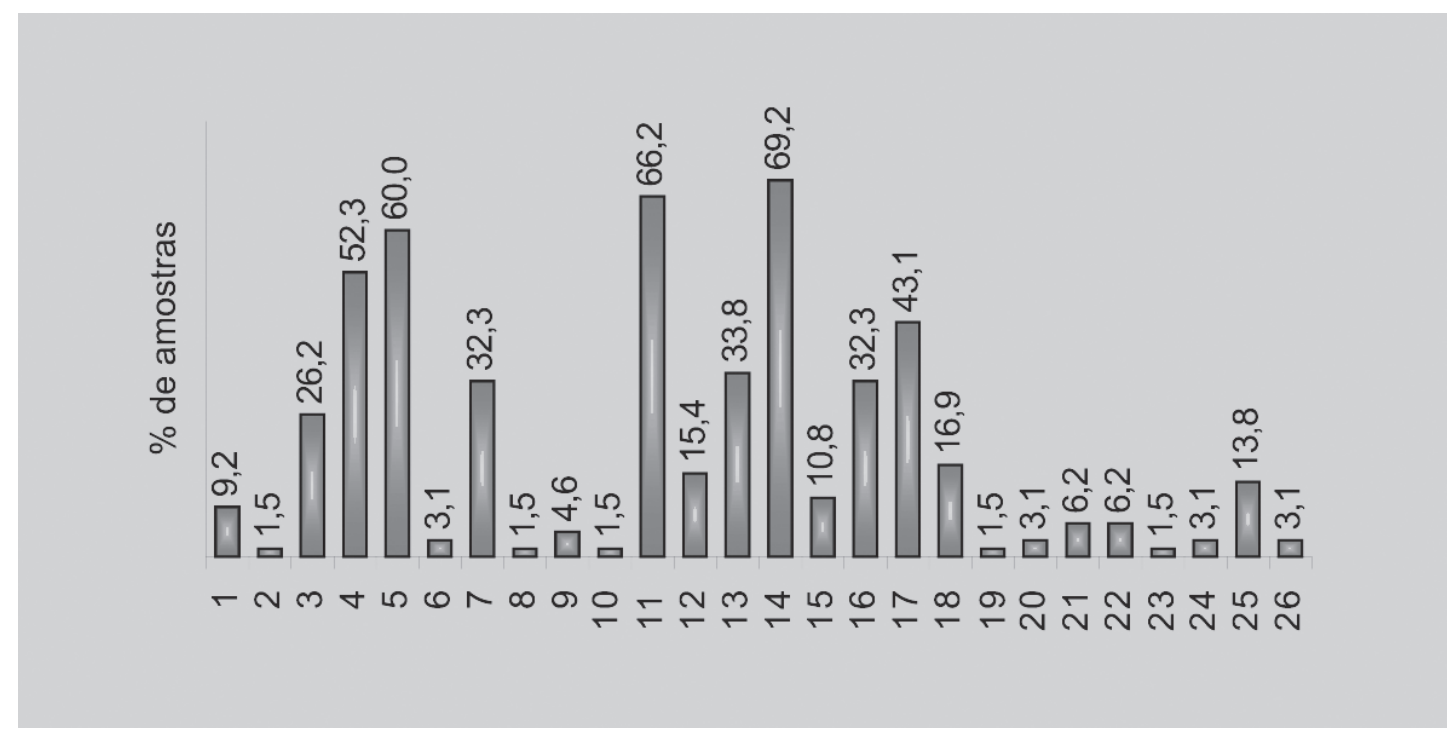

FIGURA 2 - Porcentagem de drogas vegetais que apresentaram contaminação com microrganismo específico. 
o detectaram em $49,4 \%, 75,4 \%$ e $96,8 \%$ das amostras analisadas, respectivamente, sendo que Martins et al. (2001) verificaram contagens superiores a $10^{3} \mathrm{UFC} / \mathrm{g}$ em $19,2 \%$ das amostras.

Embora as especificações farmacopéicas indiquem somente a pesquisa de Aspergillus flavus e Aspergillus parasiticus, devido à preocupação com possível ocorrência de aflatoxinas, verificou-se a presença de outros gêneros e espécies fúngicas conhecidas por sua capacidade em produzir micotoxinas, como Aspergillus niger, Aspergillus ochraceus, Aspergillus fumigatus e outros Aspergillus spp, detectados em $69,2 \%, 33,8 \%, 10,8 \%$ e $32,3 \%$ das espécies vegetais, além de Penicillium citrinum, Penicillium chrysogenum, Trichoderma spp e Alternaria spp, em $43,1 \%, 16,9 \%, 3,1 \%$ e $1,5 \%$ das espécies vegetais.

Verificou-se que os gêneros Aspergillus e Penicillium foram detectados com maior frequência, em conformidade com os resultados obtidos Abou-Arab et al. (1999), Aziz et al. (1998) e El-Kady et al. (1992), que avaliaram amostras de drogas vegetais e fitoterápicos disponíveis no Egito, Chourasia (1995), na Índia, Efuntoye (1996), na Nigéria, Elshafie et al. (1999, 2002), no Sultanato de Oman, Garrido et al. (1992), na Espanha, Halt (1998), na Croacia, Lutomski e Kedzia (1980), na Polônia, Mandeel (2005) em Bahrain, Martins et al. (2001) em Portugal, Rizzo et al. (2004) na Argentina e Santos et al. (1995), no Brasil.

Os resultados obtidos evidenciaram que $81,5 \%$ das drogas vegetais analisadas podem ser consideradas insatisfatórias por apresentarem pelo menos uma das espécies consideradas como indicadores de risco. Entretanto, considerandas as especificações farmacopéicas para a enumeração de populações microbianas presentes e para pesquisa de microrganismos específicos, verificou-se que $92,3 \%$ das drogas vegetais analisadas estavam em desacordo com um ou mais parâmetros microbiológicos.

Considerando o risco real de aquisição e utilização de produtos naturais de má qualidade, é necessário garantir sua qualidade e segurança, através do controle e fiscalização rigorosas, com adoção de medidas regulamentadoras e educativas.

\section{CONCLUSÃO}

Considerando-se as especificações adotadas para a enumeração da carga microbiana presente e pesquisa de microrganismos específicos, verificou-se que $92,3 \%$ das espécies vegetais estão em desacordo com um ou mais parâmetros microbiológicos. Estes dados sugerem que drogas vegetais podem ser consideradas produtos de alto risco, sendo necessário definir medidas adequadas de controle higiênico-sanitário para garantir a qualidade e segu- rança deste tipo de produto desde a coleta, armazeamento e manipulação até o produto final.

\section{ABSTRACT}

\section{Evaluation of microbiological contamination of crude herbal drugs}

The increasing consumption of natural drugs has made their use a Public Health issue, due to the possibility of accessing products without quality. The concern about quality is mainly due to the potential microbiological contamination of the products, by their natural origin. Ninety-one samples of sixty-five herbal species were evaluated in relation to microbial contamination. Results indicated that $92.3 \%$ of the herbal drugs failed to comply with the pharmacopeial parameters of acceptance and therefore, it suggests that regulatory and educational measures are needed in order to guarantee the quality of these products.

UNITERMS: Herbal drugs. Quality control. Microbiological contamination.

\section{REFERÊNCIAS BIBLIOGRÁFICAS}

ABOU-ARAB, A. A. K; KAWTHER, M. S.; EL TANTAWY, M. E.; BADEAA, R. I.; KHAYRIA, N. Quantity estimation of some contaminants in commonly used medicinal plants in the Egyptian market. Food Chem., Barking, v. 67, p. 357-363, 1999.

ABU-IRMAILEH, B. E.; AFIFI, F. U. Herbal medicine in Jordan with special emphasis on commonly used herbs. J. Ethnopharmacol., Lausanne, v. 89, p. 193-197, 2003.

ALEXANDER, R. G.; WILSON, D. A.; DAVIDSON, A. G. Medicines control agency investigation of the microbial quality of herbal products. Pharm. J. Verwoodburg, v. 259, p. 259-261, 1997.

AZIZ, N. H.; YOUSSEF, Y. A.; EL-FOULY, M. Z.; MOUSSA, L. A. Contamination of some common medicinal plant samples and spices by fungi and their mycotoxins. Bot. Bull. Acad. Sinica, Cairo, v. 39, n. 4, p. 279-285, 1998.

BENT, S.; KO, R. Commonly used herbal medicines in the United States: a review. Am. J. Med., New York, v. 116, p. 478-485, 2004. 
BERMUDEZ, A. D. M.; LECEA, J. R.; SOLIS, F. M.; ESTERAS, T. S. Micoflora de matérias primas. Boll. Chim. Farm., Milano, v. 123, p. 123-129, 1983.

BRASIL. Resolução RDC n ${ }^{\circ}$ 48, de 16 de março de 2004. Dispõe sobre o registro de medicamentos fitoterápicos. Diário Oficial [da] República Federativa do Brasil, Brasília, DF, 18 mar. 2004. Disponível em: <http:// www.anvisa.gov.br/legis/resol/2004/48_04rdc.htm>. Acesso em: 22 mar. 2004.

CALIXTO, J. B. Efficacy, safety, quality control, marketing and regulatory guidelines for herbal medicines (phytotherapeutic agents). Braz. J. Med. Biol. Res., Ribeirão Preto, v. 33, p. 179-189, 2000.

CAPASSO, F. The medicinal plants in our time. Boll. Chim. Farm., Milano, v. 125, n. 9, p. 322-327, 1986.

CHAN, K. Some aspects of toxic contaminants in herbal medicines. Chemosphere, Oxford, v. 52, p. 1361-1371, 2003.

CHOURASIA, H. K. Mycobiota and mycotoxins in herbal drugs of Indian pharmaceutical industries. Mycol. Res., Cambridge, v. 99, n. 6, p. 697-703, 1995.

CZECH, E.; KNEIFEL, W.; KOPP, B. Microbiological status of commercially available medicinal herbal drugs - a screening study. Planta Med., Stuttgart, v. 67, p. 263-269, 2001.

DE SMET, P. A. G. M. Health risks of herbal remedies: an update. Clin. Pharmacol. Ther., St. Louis, v. 76, p. 1-17, 2004.

EFUNTOYE, M. O. Fungi associated with herbal drug plants during storage. Mycopathologia, Den Haag, v. 136, p. 115-118, 1996.

EL-KADY, I. A.; EL-MARAGHY, S. S. M.; MOSTAFA, E. Contribution of the mesophilic fungi of different spices in Egypt. Mycopathologia, Den Haag, v. 120, p. 93-101, 1992.

ELSHAFIE, A. E.; AL-LAWATIA, T.; AL-BAHRY, S. Fungi associated with black tea and tea quality in the Sultanate of Oman. Mycopathologia, Den Haag, v. 145, p. 89-93, 1999.
ELSHAFIE, A. E.; AL-RASHDI, T.; AL-BAHRY, S. N.; BAKHEIT, C. S. Fungi and aflatoxins ssociated with spices in the Sultanate of Oman. Mycopathogia, Den Haag, v. 155, p. 155-160, 2002.

ELVIN-LEWIS, M. Should we be concerned about herbal remedies? J. Ethnopharmacol., Clare, v. 75, p. 141-164, 2001.

FARMACOPÉIA Brasileira. 4. ed. São Paulo: Atheneu, 1988, Parte 1. p. V.5.1.6.-1 - V.5.1.7.-6.

FENNELL, C. W.; LIGHT, M. E.; SPARG, S. G; STAFFORD, G. I.; VAN STADEN, J. Assessing African medicinal plants for efficacy and safety: agricultural and storage practices. J. Ethnopharmacol., Clare, v. 95, p. 113-121, 2004.

FISCHER, D. C. H.; OHARA, M. T.; SAITO, T. Contaminação microbiana em medicamentos fitoterápicos sob a forma sólida. Rev. Farm. Bioquim. Univ. S. Paulo, São Paulo, v. 29, p. 81-88, 1993.

FISCHER, D. C. H.; OHARA, M. T.; SAITO, T. Padrão microbiano em medicamentos não estéreis de uso oral. Rev. Bras. Farmacogn., São Paulo, v. 1, p. 29-54, 1996.

GARCIA, E. S.; SILVA, A. C. P.; GILBERT, B.; CORREA, C. B. V.; CAVALHEIRO, M. V. S.; SANTOS, R. R. E TOMASSINI, T. Biodiversidade: perspectivas e oportunidades tecnológicas. Fitoterápicos. Disponível em : <http://www.bdt.org.br/publicações/padct/bio/ cap10/Eloi.html>. Acesso em: 12 fev. 2003.

GARRIDO, D.; JODRAL, M.; POZO, R. Mold flora and aflatoxin-producing strains of Aspergillus flavus in spices and herbs. J. Food Prot., Des Moines, v. 55, n. 6, p. 451452, 1992.

GIVEON, S.M.; LIBERMAN, N.; KLANG, S.; KAHAN, E. Are people who use "natural drugs" aware of their potentially harmful side effects and reporting to family physician? Patient Educ. Couns., Princeton, v. 53, p. 511, 2004.

HALT, M. Moulds and mycotoxins in herb tea and medicinal plants. Eur. J. Epidemiol., New York, v. 14, p. 269-274, 1998. 
KNEIFEL, W.; BERGER, E. Microbiological criteria of random samples of spices and herbs retailed on the Austrian market. J. Food Prot., Des Moines, v. 57, n. 10, p. 893-901, 1994.

KOROLKOVAS, A. A riqueza potencial de nossa flora. Rev. Bras. Farmacognosia, São Paulo, v. 1, p. 1-7, 1996.

LUTOMSKI, J.; KEDZIA, B. Mycoflora of crude drugs. Estimation of mould contaminations and their toxicity. Planta Medica, Stuttgart, v. 40, p. 212-217, 1980.

MAHADY, G. B. Global Harmonization of Herbal Health Claims. J. Nutr., Philadelphia, v. 131, p. 1120S-1123S, 2001.

MANDEEL, Q. A. Fungal contamination of some imported spices. Mycopathologia, Den Haag, v. 159, p. 291-298, 2005

MARQUES, L. C. Vigilância de fitoterápicos - I: o caso da arnica. Rev. Bras. Farmacogn., São Paulo, v. 1, p. 8-19, 1996.

MARTINS, H. M.; MARTINS, M. L.; DIAS, M. I.; BERNARDO, F. Evaluation of microbiological quality of medicinal plants used in natural infusions. Int. J. Food Microbiol., Amsterdam, v. 68. p. 149-153, 2001.

PITT, J. I. The genus Penicillium. Sidney, Australia: Academic Press Inc., 1979.
RAPPER, K. B.; FENNEL, D.I. The genus Aspergillus. Baltimore: The Williams and Wilkins Company, 1965.

RATES, S. M. K. Plants as source of drugs. Toxicon, v. 39, p. 603-613, 2001.

RHODEHAMEL, E.J.; HARMON, S.M. Bacillus cereus. In: Bacteriological Analytical Manual Online, 2001. FOOD AND DRUGS ADMINISTRATION - Center for Food Safety and Applied Nutrition. Disponível em: $<\mathrm{http}: / /$ www.cfsan.fda.gov/>. Acesso em: 10 jan. 2003.

RIZZO, I.; VEDOYA, G.; MAURUTTO, S.; HAIDUKOWSKI, M.; VARSAVSKY, E. Assessment of toxigenic fungi on Argentinean medicinal herbs. Microbiol. Res., Buenos Aires, v. 159, n. 2, p. 113-120, 2004.

SANTOS, P. R. V.; OLIVEIRA, A. C. X.; TOMASSINI, T. C. B. Controle microbiológico de produtos fitoterápicos. Rev. Farm. Bioquim. Univ. S. Paulo, São Paulo, v. 31, p. 35-38, 1995.

THE UNITED States Pharmacopeia. 28. ed. Rockville: United States Pharmacopeial Convention, 2005.3013 p.

Recebido para publicação em 14 de dezembro de 2004. Aceito para publicação em 24 de novembro de 2005. 